\title{
Experiencia de libre albedrío en la conducta pro-social determinada por la alegría
}

\section{The experience of free will in pro-social behaviour mediated by happiness}

\author{
Joaquim Coma y Xavier Carbonell \\ Universitat Ramon Llull, FPCEE Blanquerna, España \\ Disponible online 31 de diciembre de 2015
}

\begin{abstract}
Isen y Levin (1972) determinaron la conducta pro-social mediante la alegría. En este estudio se ha replicado el experimento de Isen y Levin en una muestra de 127 personas, introduciendo algunos cambios para adaptarlo al contexto actual. Se ha registrado la experiencia de libertad de los participantes y se han relacionado los resultados con la explicación de construcción de creencias de Gazzaniga. Se ha corroborado la hipótesis de Isen y Levin en el contexto actual y la experiencia de libre albedrío en los participantes.

Palabras Clave: Libertad; Determinismo; Altruismo.

Isen and Levin (1972) suggested that pro-social behaviour was mediated by happiness. This study replicated the experiment conducted by Isen and Levin in a sample of 127 people, introducing some changes to adapt it to the current settings. The experience of freedom of the participants was recorded and the results related to Gazzaniga's construction of belief. The hypothesis provided by Isen and Levin was upheld in the current setting and the experience of free will in the participants.
\end{abstract}

Key Words: Freedom; Determinism; Altruism. 
Psicólogos y filósofos debaten desde hace tiempo la naturaleza y la existencia del libre albedrío. Existen dos puntos de vista principales y opuestos. Los que afirman que gozamos de libre albedrío opinan que algún factor $x$ nos permite tomar decisiones o determinar nuestras acciones, e incluso nuestro destino, interviniendo e introduciendo cambios en el mundo físico. Los que no aceptan el libre albedrío creen que vivimos en un mundo predeterminado en el que toda acción, sea humana o no lo sea, es inevitable (Gazzaniga, 2006).

El filósofo y neurocientífico Sammuel Harris (2012) revisó los estudios defensores del determinismo. Entre ellos, Libet (1985) utilizó el electroencefalograma para mostrar que la actividad en la corteza motora del cerebro puede ser detectada unos 300 milisegundos antes que la persona piense que ha decidido moverse. Otro laboratorio extendió este trabajo utilizando imágenes de resonancia magnética funcional: se les pidió a los sujetos que pulsaran un botón de dos posibles mientras miraban un reloj compuesto por una secuencia aleatoria de letras que aparecían en una pantalla. Estos sujetos tenían que decir qué letra era visible en el momento en que decidiesen pulsar un botón u otro. Los experimentadores encontraron dos regiones cerebrales que contienen información sobre qué botón pulsarían los sujetos en un total de siete a diez segundos antes que la decisión fuera tomada conscientemente. Más recientemente registros directos de la corteza cerebral mostraron que la actividad de solamente 256 neuronas era suficiente para predecir, con un 80 por ciento de precisión, la decisión de una persona de moverse 700 milisegundos antes de que empezara a ser consciente de ello. Harris (2012) afirma que "estos descubrimientos son difíciles de reconciliar con la sensación de que nosotros somos los autores conscientes de nuestras propias acciones" (p.9). Según Harris (2012) es indiscutible que "unos instantes antes de que seas consciente de qué piensas hacer después, tiempo en el que tú, subjetivamente, crees tener la completa libertad de comportarte como desees, tu cerebro ya ha determinado qué es lo que harás" (p. 9). Entonces es cuando se toma consciencia de esta 'decisión' y la persona cree que ha formado parte del proceso de esta 'decisión'. Como razona Gazzaniga (2006, 2012), muchos experimentos recalcan la idea de que el cerebro hace cosas antes de que seamos conscientes de ello. Estos datos parecen favorecer la idea de que el libre albedrío es más una ilusión que una realidad. Por otro lado, el mismo Gazzaniga $(2006,2012)$, considera que probablemente los cerebros sean automáticos pero las personas seamos libres.

Brasil, Pascual, Valls, Cohen y Hallet (1992) no pretendían mostrar si el ser humano puede o no tomar decisiones libremente, sino estudiar cómo es la percepción consciente de una situación determinada. Para hacerlo, determinaron la conducta de los participantes utilizando la Estimulación Magnética Transcraneal (EMT) en el área motora del cerebro y se les pedía que escogiesen mover el dedo índice derecho o izquierdo. Aunque movieron el dedo contra-lateral al lado cerebral estimulado por la EMT, los participantes reportaron sentir que escogían voluntariamente el dedo que movieron.
Para estudiar la percepción que las personas pueden tener de su supuesto libre albedrío, Stillman, Baumeister y Mele (2011) analizaron esta percepción consciente en su vida cotidiana. Encontraron que los participantes tendían a reconocerse libres en acciones relacionadas con alcanzar objetivos, niveles altos de pensamientos conscientes y deliberación, resultados positivos y comportamiento ético, entre otros.

Siguiendo la idea de que muchas operaciones del sistema motor que guían la acción resultan opacas al acceso consciente, la voluntariedad de esos actos también podría no ser consciente (Froufe, 2007). Así es como la no consciencia de la causa de la conducta en los participantes del experimento de Brasil et al. (1992) no demuestra la inexistencia de esa causa. ¿Cómo es posible que sujetos que mueven el dedo en función de la EMT sean capaces de creer que la causa de esa conducta es su propia decisión libre? En palabras de Froufe (2007), la experiencia de voluntad intencional puede proceder con independencia de las fuerzas causales reales de la conducta.

Gazzaniga (2006) defiende así su idea sobre la naturaleza de las creencias: nuestra especie reacciona de manera instintiva a los acontecimientos y en un sistema integrado del cerebro humano se interpreta esta reacción. De esa interpretación nacen las creencias sobre las reglas que deben regir nuestra vida. En la mayoría de casos son interpretaciones basadas en el conocimiento disponible en el momento en que se van a formar, y que, no obstante, parecen quedar grabadas en la mente. Este autor llama intérprete a esta zona del hemisferio izquierdo porque busca explicaciones para los sucesos internos y externos, y amplía los hechos reales que experimentamos con la finalidad de comprender o interpretar los sucesos de la vida. Varios estudios de Gazzaniga (1993, 2006, 2012) con pacientes con escisión cerebral revelan la facilidad con la que el intérprete del hemisferio izquierdo inventa para poder sustentar sus creencias. Un ejemplo de estos estudios es cuando presentó a los pacientes con escisión cerebral la palabra "camina" solo al lado derecho del cerebro de un paciente, este se levantó y se puso a caminar. Cuando se le preguntó por qué lo había hecho, el cerebro izquierdo (donde se almacena el lenguaje y donde la palabra "camina" no había sido presentada) se inventó rápidamente un motivo para justificar su acción: "quería ir a buscar una Coca-Cola". El intérprete del hemisferio izquierdo, continúa Gazzaniga (2006), no solo es un maestro de la elaboración de creencias, sino que se apega a su sistema de convicciones pase lo que pase. El intérprete hace todo lo posible para asegurarse que las informaciones que recibe queden atadas de tal manera que tengan sentido, aunque para eso tenga que hacer "malabarismos". La persona, por supuesto, no lo percibe como "malabarismos", sino como claras manifestaciones del mundo circundante (Gazzaniga, 2006). El intérprete es un módulo que explica los acontecimientos a partir de la información que recibe, que son los resultados de las computaciones de multitud de módulos (Gazzaniga, 2012). 
Relacionándolo, ahora, con el concepto de libre albedrío, el intérprete ha creado la ilusión del yo y, con ella, el sentido de que los seres humanos tenemos agentividad y tomamos decisiones libres que determinan nuestra acción. El intérprete aporta la línea argumental y el relato; y todos nos creemos que somos agentes y actuamos en función de nuestro libre albedrio, tomando decisiones importantes. La ilusión es tan profunda que no hay ningún análisis capaz de cambiar la sensación de que actuamos de forma volitiva y deliberada. (Gazzaniga, 2012)

Siguiendo con la aproximación a la relación entre libre albedrío y determinismo, Muñoz (2013), desde su perspectiva neuro-filosófica, analiza los estudios psicológicos y neurológicos recientes relacionados con las bases biológicas del proceso volitivo. Para este autor, la posición más prudente sería pensar que, si bien ciertas áreas resultan decisivas en la generación de nuestras decisiones voluntarias (en especial la corteza prefrontal), estas áreas no podrían ejercer esta función si no estuvieran integradas en un amplio sistema formado por diversas estructuras, en el cual la cognición, la memoria y la emoción están estrechamente interrelacionadas. También en el sistema de control atencional se integran distintas áreas cerebrales, como el lóbulo parietal, la corteza temporal, la corteza frontal y estructuras subcorticales (Gazzaniga, Ivry y Mangun, 2009). Esta integración ha sido estudiada también en la fuerte influencia que tiene la corteza orbitofrontal (que conecta con las áreas cerebrales relacionadas con las emociones) en la aplicación del conocimiento social al tomar decisiones en este contexto (Beer, 2009). Cada vez parece más evidente, concluye Gazzaniga (2012), que la consciencia conlleva multitud de procesos desunidos, cuyos resultados se integran de manera dinámica mediante el módulo intérprete del hemisferio izquierdo.

Centrándonos, ahora, en el concepto de emoción, Torregrosa (1984) argumenta la concepción de la emoción como un estado de consciencia que no solo refleja la excitación, sino también otros contenidos de naturaleza cognoscitiva. Es decir, en la medida en que las emociones se vinculan a la experiencia concreta de seres humanos socializados, por definición miembros de una cultura, las emociones estarán mediatizadas por procesos cognitivos y evaluativos; y por eso serán, en gran medida, una producción social, el resultado de procesos sociales de interacción y del marco sociocultural en el que esta interacción suceda.

El experimento seminal de Isen y Levin (1972) ejemplifica cómo la emoción puede ser un determinante de la conducta. El experimento pretendía evaluar los efectos de la emoción en la actitud pro-social de los participantes. Colocaron una moneda en una cabina telefónica y evaluaron si encontrarla influía en la respuesta del participante de ayudar o no a recoger unos papeles que le caían a una segunda persona, colaborador del experimentador. Así la moneda era el estímulo que provocaba la emoción de alegría en los participantes, y la respuesta de ayuda en recoger los papeles era la forma de registrar la actitud pro-social. El $87.5 \%$ de las personas que encontraron la moneda en la cabina telefónica proporcionaron la ayuda recogiendo los papeles del suelo. Solo el $4 \%$ de los participantes que no encontraron la moneda acudieron a prestar su ayuda.

Tomando como base distintas premisas de autores antes citados, los sujetos del experimento de Isen y Levin (1972) no estarían siendo determinados sólo por la emoción, sino por la conjunción entre la emoción y otras estructuras neuronales que forman parte de un sistema integrado (Muñoz, $2013 \mathrm{y}$ Gazzaniga, et al. 2009) y por los constructos socioculturales de producción social (Torregrossa, 1984). En el experimento, sin embargo, solo se estaría controlando la emoción y no otros elementos también influyentes en el proceso de determinación de la conducta.

Como expone Garrido (2000), desde el modelo de acción humana, se puede afirmar que los diferentes niveles de emoción están estrechamente vinculados con los niveles de actividad, siendo la emoción una forma de acción que integra tres niveles de actividad que se concretan en tres componentes: experiencia, cambios neurofisiológicos y endocrinos y expresión emocional. Según este mismo autor, la emoción influye en la acción a través de una serie de mecanismos que están estrechamente vinculados a la funcionalidad de la emoción. Además, la relación entre estas variables de alegría y actitud pro-social puede ser también inversa: las personas que gastan dinero en otras personas reportan más felicidad (Dunn, Aknin y Norton, 2014). Los mismos autores ya demostraron que la relación causal entre estas variables era circular (Aknin, Dunn y Norton, 2011). Los estudios de Aknin et al. (2013) sugieren que la recompensa experimentada al ayudar a otras personas puede estar fuertemente arraigada a la naturaleza humana, ya que relacionaron las variables en participantes de 136 países y en diferentes contextos económicos.

Godman (2013) se ha interesado por la motivación social compartida como una causa significativa de la mayoría de las acciones conjuntas. Las motivaciones sociales, consideradas como un grupo de factores emocionales y afectivos, se distinguen de otros afectos y emociones precisamente por su carácter pro-social. Esto es, las emociones relevantes son provocadas específicamente en asuntos sociales que involucran a otras persones y éstas hacen de la experiencia social una recompensa para ellos mismos.

En referencia a este concepto de actitud pro-social, la posición biológica es que, así como los seres humanos tienen tendencias innatas a beber y comer, también tienen tendencias a ayudar a los otros. Hay casos de cooperación también en el comportamiento animal. Un ejemplo es el de las ardillas, que dan la señal de alarma al resto del grupo, aunque eso signifique llamar la atención de los depredadores de su propia presencia. Casos como éste indican que la adaptación evolutiva ha promovido la conducta cooperativa en los animales y en los humanos (Hogg y Vaughan, 2010). A pesar de esta disposición del ser humano a actuar de manera pro-social, hay que considerar que distintos factores individuales son influyentes en la conducta pro-social. Por ejemplo, Aghababaei, Mohammadtabar y Saffarinia (2014) mostraron la influencia de la humildad y la hones- 
tidad, como factor individual, en la actitud pro-social del individuo. Aun así, es necesario tener presente que la evolución creó el cerebro humano como herramienta de supervivencia y procreación; los sentimientos están, consecuentemente, destinados a promover el comportamiento más apropiado para sostener la vida, no para hacer esta vida más placentera (Grinde, 2002). Los vertebrados están presumiblemente equipados con sensaciones designadas a inducirlos a comportarse, es decir, a tomar decisiones, conduciéndolos a propagar sus genes. El comportamiento consiste generalmente en "hacer lo mejor que ofrezca la oportunidad" o, en cambio, "evitar alguna cosa"; dos maneras de comportarse que corresponden a las sensaciones positivas y negativas (Nesse y Williams, 1996). Esto, argumenta Grinde, conduce a pensar que las sensaciones positivas y negativas tienen un mayor impacto en la adaptación de los humanos que en otras especies. Presumiblemente, el experimento de Isen y Levin (1972) es una explicación de cómo una emoción, en este caso positiva, es causante de un comportamiento positivo para la especie, la actitud pro-social.

Tomando como punto de partida estos argumentos, este trabajo pretende, en primer lugar, comprobar si la réplica del experimento de Isen y Levin (1972), en un nuevo contexto, con más de cuarenta años de diferencia y en un país diferente, continúa demostrando que la emoción de alegría, motivada por un obsequio inesperado, puede ser un determinante de la respuesta de los sujetos cuando se les ofrezca la oportunidad de realizar una conducta pro-social. Se ha evidenciado que hay múltiples experimentos neuropsicológicos que demuestran que la experiencia de libre albedrío puede proceder aun con la existencia, no percibida conscientemente, de una fuerza causal real. Así pues, en segundo lugar, este trabajo pretende comprobar si, con la réplica del experimento de Isen y Levin, los participantes comunican sentirse libres en la toma de la decisión aunque exista un proceso real de determinación de la conducta. Hemos presentado, en palabras de Gazzaniga (2006), cómo el ser humano tiende a aferrarse a las creencias que se ha formado con la información disponible. El tercer objetivo de este estudio es si dada una argumentación racional a los participantes de que han estado en una situación en que su conducta ha sido determinada, se mantendrán en la creencia de que su conducta ha sido consecuencia de una decisión tomada libremente y relacionar esos resultados con la teoría expuesta por Gazzaniga $(2006,2012)$.

Método
Participantes
Participaron un total de 127 personas. La edad de los par-
ticipantes se categorizó en tres grupos, en función del criterio
de los experimentadores: menos de treinta años, entre 30 y 60
y más de 60 . Se descartaron los participantes menores de edad.
En el grupo experimental participaron 62 personas ( 27 hombres
y 35 mujeres); 12 de los participantes menores de 30 años, 36
entre 30 y 60 y 14 mayores de 60 . Al grupo control (no recibi-
miento del obsequio) se asignaron 65 personas, 32 de las cuales

eran hombres y 33 eran mujeres; 11 participantes menores de 30 años, 35 entre 30 y 60, y 19 mayores de 60 .

\section{Procedimiento}

Los dos experimentadores (un hombre y una mujer) se situaron en una calle céntrica de la ciudad de Girona, con una calzada de anchura superior a los tres metros, próximos a un establecimiento de venta de productos de oficina y papelería. Con el grupo control, se procedió a realizar la demanda de colaboración usando la formulación: “¿Perdone, le importaría que le hiciese unas preguntas?". Los dos experimentadores se alternaron en sus posiciones.

Con el grupo experimental, el primer experimentador, situado al portal del establecimiento de productos de oficina, ofrecía un bolígrafo a las personas que pasaban por delante; solo a las que no estuvieran acompañadas y que fueran en dirección al segundo experimentador, quien haría la demanda de colaboración. Estos serían los participantes que entraron a formar parte del grupo experimental. Entonces, cuando la persona que había aceptado el obsequio se acercaba al segundo experimentador, situado a veinte metros de distancia, se le realizaba la siguiente demanda de colaboración: “¿Perdone, le importaría que le hiciera unas preguntas?". Cuando el participante no atendía a la demanda o daba alguna excusa y seguía andando, el experimentador registraba la respuesta del sujeto como "No colabora". Cuando el participante mostraba interés, haciendo alguna pregunta al respecto o respondiendo afirmativamente a la pregunta formulada, deteniendo la marcha y encarándose al experimentador, este registraba la respuesta del sujeto como "Sí colabora". Con este último grupo de participantes, los que sí colaboraron, se procedió a realizar las preguntas para el registro del segundo objetivo:

Pregunta 1. "Soy estudiante universitario, y las preguntas que le hago son para mi proyecto final de carrera. ¿Considera usted que se ha parado a colaborar conmigo porque le han regalado un bolígrafo?".

Normalmente, la primera reacción de los participantes era de sorpresa dado que no habían relacionado hasta entonces que hubiera relación entre la entrega del bolígrafo y la solicitud para responder unas preguntas. Una vez habían respondido se procedía a darles la siguiente información para el análisis del tercer objetivo:

Pregunta 2. "Estuvimos haciendo el mismo ejercicio, como con usted ahora, pidiendo a la gente si les importaría responderme a unas preguntas. Lo hicimos sin regalarles un bolígrafo. Un $80 \%$ de las personas dijeron que no, que tenían prisa o alguna otra excusa. Ahora queremos ver si a las personas que se les ha regalado un bolígrafo, como con usted, se paran más a ayudar que el otro grupo. Entonces sabremos si el bolígrafo actúa como determinante de la colaboración. Con esta información que le doy, puede contestarme otra vez la pregunta: ¿considera usted que se ha parado a colaborar conmigo porque le han regalado un bolígrafo?" 


\section{Resultados}

En la Tabla 1 se muestran los resultados del registro de la colaboración en los participantes que recibieron bolígrafo y los que no.

Tabla1

Relación entre la alegría y la colaboración.

\begin{tabular}{cccc}
\hline \multirow{2}{*}{} & & \multicolumn{2}{c}{ Colaboración } \\
\cline { 2 - 4 } & & $\begin{array}{c}\text { Acepta } \\
n(\%)\end{array}$ & $\begin{array}{c}\text { No acepta } \\
n(\%)\end{array}$ \\
\hline \multirow{2}{*}{ Bolígrafo } & Sí $(n=56)$ & $29(51.8 \%)$ & $27(48.2 \%)$ \\
\cline { 2 - 4 } & No $(n=65)$ & $14(21.5 \%)$ & $51(78.5 \%)$ \\
\hline
\end{tabular}

Los resultados del Chi-cuadrado de Pearson $\left(x^{2}\right)$ dan un valor 12.014 y una $p=.001$ lo que permite afirmar que ser obsequiado con un bolígrafo y prestar colaboración son variables relacionadas. En la tabla 2 se muestran las respuestas a los 29 participantes que sí habían sido obsequiados y que sí se habían prestado a responder las preguntas.

Tabla 2

Respuestas a la pregunta "¿Considera usted que se ha parado a colaborar conmigo por qué le han regalado un bolígrafo?"

\begin{tabular}{ccccc}
\hline & \multicolumn{2}{c}{$\begin{array}{c}\text { Pregunta 1: antes de dar } \\
\text { información }\end{array}$} & \multicolumn{2}{c}{$\begin{array}{c}\text { Pregunta 2: después de dar } \\
\text { información }\end{array}$} \\
\cline { 2 - 5 } & No & Sí & No & Sí \\
\hline $\begin{array}{c}\text { Participantes } \\
(\mathrm{n}=29)\end{array}$ & $29(100 \%)$ & $0(0 \%)$ & $25(86.2 \%)$ & $4(13.8 \%)$ \\
\hline
\end{tabular}

\section{Discusión}

Este experimento corrobora que la emoción es un condicionante de la conducta y, más concretamente, que la alegría de ser obsequiado inesperadamente y sin causa aparente es causa de actitud pro-social (Isen y Levin, 1972). Isen y Levin lo demostraron obsequiando con una moneda y registrando la actitud pro-social comprobando si los sujetos ayudaban a recoger unos papeles que habían caído al suelo. Por tanto, la hipótesis de Isen y Levin (1972) se mantiene en una cultura y época bien diferentes y confirma la relación positiva entre felicidad y alegría y conducta pro-social (Javaloy, Rodríguez-Carballeira, Cornejo y Espelt, 1998). Como argumenta Grinde (2002), en los humanos las sensaciones positivas y negativas tienen un fuerte impacto en la adaptación. El experimento que hemos presentado es un buen ejemplo para demostrar la afirmación de este autor ya que la conducta pro-social es una conducta adaptativa del ser humano.

La persona que vive un estado emocional de alegría se siente complacida, embargada por el goce, contenta, en un estado de éxtasis de intensidad variable y con una disposición de ánimo eufórico, que en su manifestación extrema no se encuentra exenta de cierta exaltación y vehemencia. Estos sentimientos promueven una actitud optimista en el afrontamiento de las vicisitudes que se plantean en la vida cuotidiana y favorecen el incremento de la autoestima y de la confianza en las propias capacidades (Fernández, Jiménez y Martín, 2003).
A diferencia del miedo o de la ansiedad, la alegría no encuentra su funcionalidad en la protección del ser humano ante posibles amenazas; la funcionalidad de la alegría se ha de buscar, pues, en los efectos que esta emoción genera en la regulación de los sistemas biológicos y psicológicos de la persona, y en las funciones que desarrolla en la interacción de ésta con su medio natural (Fernández et al., 2003). Los mismos autores exponen que la alegría podría actuar como una emoción que atenúa la respuesta fisiológica al estrés o agilizando el reajuste homeostático del organismo después de afrontar una situación estresante. La expresión alegre, continúan Fernández et al. (2003), informa a un eventual interlocutor de nuestra buena disposición para iniciar una relación interpersonal o comunicativa. Esta relación de las variables fue estudiada también por Javaloy et al., (1998), ratificando que un estado de ánimo jovial favorece nuestra disposición para desarrollar conductas pro-sociales.

De todas formas, permaneciendo en la línea de Muñoz (2013) y Torregrossa (1984), la emoción no puede ser el único determinante de un proceso volitivo, sino que este proceso es determinado también por otras dimensiones que afectan a nuestro pensamiento y que en nuestro estudio no hemos podido registrar. Las emociones pueden estar mediatizadas por otros procesos cognitivos que, al mismo tiempo, habrán recibido la influencia pertinente de la cultura y la sociedad. En la misma dirección apunta Muñoz (2013) cuando explica que aunque hay áreas del sistema nervioso (en especial la corteza prefrontal) que resultan decisivas en el proceso de tomar decisiones voluntarias, estas áreas están integradas en un amplio sistema de estructuras neuronales relacionadas con la cognición, la emoción y la memoria, entre otras. Es así como se explica que la emoción puede no determinar siempre directamente una conducta, pero que sí forma parte de este proceso de determinación llegando a ser un elemento significativamente determinante.

Sin embargo, una parte de la muestra no ha sido determinada por la alegría. Esto no es una prueba de la existencia del libre albedrío (tampoco de la no existencia de éste), sino una prueba de que el proceso volitivo de las personas es un sistema que integra, entre otros, la cognición, la emoción y la memoria (Muñoz, 2013) y que nuestros participantes podrían estar siendo determinados por otros patrones cognitivos más influyentes que la emoción provocada por el bolígrafo. Más aún si tenemos en cuenta que en nuestro estudio solo se manipuló una de estas partes del sistema volitivo, la emoción. Aquí simplemente se ha conseguido que los participantes del grupo experimental estuvieran más alegres que los del grupo control y que esta alteración influenciara en la conducta.

En referencia a la segunda hipótesis, hemos comprobado que a pesar de que los participantes reportaban sentirse libres en su decisión de ayudar, su conducta estaba determinada por la alegría. Froufe (2007) afirma que sentirse libres puede proceder con independencia de las fuerzas causales reales de la conducta. Los resultados de nuestro experimento se pueden añadir a los de Brasil et al. (1992) en el que los sujetos reportaban mover 
el dedo libremente, cuando en realidad era el experimentador quien lo decidía. La diferencia entre el estudio de Brasil et al. (1992) y el nuestro es que en aquél la conducta de los participantes se manipulaba en un contexto experimental que nunca se podría dar en la vida cotidiana. Aquí, en cambio, se muestra en una situación cotidiana que nuestras emociones pueden ser determinantes inconscientes de nuestras decisiones, mientras que nuestra experiencia consciente es que disponemos de libre albedrío para tomar estas decisiones.

Como se ha explicado, en este estudio se registró la experiencia consciente de los participantes con una sencilla pregunta. Ninguno de los sujetos reconoció que su conducta había sido determinada por el obsequio que habían recibido anteriormente. Por tanto, la experiencia de voluntad intencional puede proceder con independencia de las fuerzas causales reales de la conducta (Froufe, 2007). Además, nuestra forma de preguntarlo estaba haciendo referencia a la que estaría siendo (en un numero estadísticamente significativo de participantes) la fuerza causal real, es decir, el bolígrafo. En nuestro experimento, la supuesta acción libre que sentían los participantes hacía referencia a un comportamiento pro-social, considerado como positivo. Esta experiencia de libertad podría venir marcada por el hecho de que las personas tienden a sentirse libres cuando la acción se relaciona con, entre otros, el comportamiento ético y los resultados positivos (Stillman et al., 2011).

La información recogida en este estudio no se detuvo con el registro de la experiencia consciente bajo la influencia de la alegría, sino que dimos a esos participantes más información sobre el experimento y su proceso de determinación. Así, se informó de que solo un $20 \%$ de los sujetos a quienes no se les había obsequiado con el bolígrafo se habían parado a ayudar. Con esta información, si se estuviera considerando la cuestión (es decir, la pregunta que se les formulaba) en términos de máxima racionalidad, deberían plantearse que, para no haber estado bajo la influencia del bolígrafo (es decir, para responder 'No'), estos tendrían que formar parte de ese $20 \%$, cosa menos probable que estar dentro del $80 \%$ que se habría parado. Disponiendo de esta información se les repitió la misma pregunta y tan solo 4 de los 29 sujetos consideró la posibilidad de que realmente la alegría provocada por el bolígrafo podría influir en su decisión. Por tanto, usamos estos resultados para sustentar la hipótesis de que la creencia de los sujetos en su libre albedrío impide hacer uso de la información racional en el análisis de la situación. En otras palabras, las personas tienden a aferrarse a su sentimiento de libertad antes que a hacer uso de la razón.

Para entender esto utilizaremos la teoría del intérprete del hemisferio izquierdo de Gazzaniga $(2006,2012)$. Como comentábamos, Gazzaniga $(2006,2012)$ explica que nuestra especie reacciona de forma instintiva ante los sucesos. Esta manera instintiva, es decir, el procedimiento automático del cerebro es, en el experimento aquí presentado, la colaboración; puesto que es la reacción automática del cerebro la que hace que la alegría sea causa de actitud pro-social. De la interpretación de la reacción, explica Gazzaniga (2006, 2012), se originan las creencias sobre las reglas que deben regir nuestra vida. Esta interpretación es en este estudio la ilusión del libre albedrío, tal como respondían los participantes a la "pregunta 1". Como continúa el mismo autor, en la mayoría de los casos son interpretaciones basadas en el conocimiento disponible en el momento en que se forman y que, no obstante, parecen quedar grabadas en nuestra mente. Los participantes del experimento no tenían la información de que la alegría es causa de actitud pro-social. Como afirma este autor, el intérprete del hemisferio izquierdo hace todo lo posible para asegurarse de que las informaciones que recibe quedan atadas de tal manera que cobren sentido, aunque para ello haya que hacer "malabarismos". Así, cuando a los participantes del experimento se les explicaba que tan solo un $20 \%$ de participantes habían colaborado cuando no habían sido obsequiados con el bolígrafo (dato que muestra que la probabilidad de que el bolígrafo haya influido es mayor), éstos no eran capaces de librarse de la creencia formada antes de recibir esa información. Este hecho podría significar que estos 'malabarismos' del hemisferio izquierdo eran, en este caso, no hacer uso de lo que la lógica afirmaría; es decir, que no se había tomado esa decisión libremente. Así es como aquí relacionamos los resultados de este estudio con la afirmación de Gazzaniga (2012) de que es el intérprete el que crea la ilusión de que poseemos agentividad y tomamos decisiones libres que determinan nuestra acción.

Otros investigadores (Aknin, et al., 2011; Dunn et al., 2014; Oerlemans y Bakker, 2014) han estudiado la relación de las variables alegría y actitud pro-social con la dirección causal inversa a la estudiada aquí; es decir, si la actitud prosocial causa felicidad. Nuestro diseño experimental permitiría avanzar también en esta dirección si se añadiese un registro de la alegría después de prestar su colaboración al experimentador.

Dado que en los humanos, por definición miembros de una cultura, los procesos cognitivos y evaluativos son, en gran parte, una producción social (Torregrossa, 1984), consideramos que una limitación de este estudio es que no se ha tenido en cuenta la concepción que tiene la cultura occidental sobre el concepto de libre albedrío y determinismo. Probablemente otras culturas tengan una concepción distinta sobre este debate, y por tanto los resultados podrían diferir en función del espacio cultural donde se aplicara este estudio. Otra posible limitación es que la argumentación racional antes de repetir la "pregunta 2" podría ser demasiado compleja para que los participantes la integrasen en el contexto en el que se encontraban. Por eso, en futuras líneas de investigación se debería comprobar si es posible integrar los datos lógicos ofrecidos antes de repetir la pregunta o diseñar otro modo de dar esa información lógica, de modo que se pueda garantizar que estos están comprendiendo esa información. 


\section{Referencias}

1. Aghababaei, N., Mohammadtabar, S. y Saffarinia, M. (2014). Dirty Dozen vs. the H factor: Comparison of the Dark Triad and Honesty-Humility in prosociality, religiosity, and happiness. Personality and Individual Differences, 67, 6-10. http://dx.doi.org/10.1016/j.paid.2014.03.026

2. Aknin, L., Dunn, E. y Norton, M. (2011). Happiness runs in a circular motion: Evidence for a positive feedback loop between prosocial spending and happiness. Journal of Happiness Studies, 13, 347-355. http://dx.doi.org/10.1007/ $\underline{\text { s10902-011-9267-5 }}$

3. Aknin, L. B., Barrington-Leigh, C. P., Dunn, E. W., Helliwell, J. F., Burns, J., Biswas-Diener, R., ... Norton, M. I. (2013). Prosocial spending and well-being: cross-cultural evidence for a psychological universal. Journal of Personality and Social Psychology, 104, 635-52. http://dx.doi. org/10.1037/a0031578

4. Beer, J. (2009). Social cognition. En M. Gazzaniga, R. Ivry y G. Mangun (Eds.), Cognitive Neuroscience: The Biology of the Mind. (pp. 599- 633). New York: Norton.

5. Brasil, J. P., Pascual, A., Valls-Solé, J., Cohen, L. G. y Hallet, M. (1992). Focal transcranial magnetic stimulation and response bias in torced-choice task. Journal of Neurology, Neurosurgery, and Psychiatry, 55, 964-966. http:// dx.doi.org/10.1136/jnnp.55.10.964

6. Dunn, E., Aknin, L. y Norton, M. (2014). Prosocial spending and happiness: Using money to benefit others pays off. Current Directions in Psychological Science, 23, 41-47. http://dx.doi.org/10.1177/0963721413512503

7. Fernández, E., Jiménez, M. y Martín, M. D. (2003). Emoción y motivación. La adaptación humana. Madrid: Centro de estudios Ramón Areces.

8. Froufe, M. (2007). Acción y conciencia. In/determinismo, autocontrol y responsabilidad. Estudios de Psicología, 28, 185-209. http://dx.doi.org/10.1174/021093907780962827

9. Garrido, I. (2000). Psicología de la emoción. Madrid: Editorial Síntesis.

10. Gazzaniga, M. (1993). El cerebro social. Madrid: Alianza Editorial.

11. Gazzaniga, M. (2006). El cerebro ético. Barcelona: Paidós.

12. Gazzaniga, M. (2012). ¿Quién manda aqui? Barcelona: Paidós.

13. Gazzaniga, M., Ivry, R. y Mangun, G. (2009). Cognitive Neuroscience: The Biology of the Mind. New York: Norton
14. Godman, M. (2013). Why we do things together: The social motivation for joint action. Philosophical Psychology, 26, 588-603. http://dx.doi.org/10.1080/09515089.2012.670905

15. Grinde, B. (2002). Happiness in the perspective of evolutionary psychology. Journal of Happiness Studies, 3, 331-354. http://dx.doi.org/10.1080/09515089.2012.670905

16. Harris, S. (2012). Free will. New York: Free Press.

17. Hogg, M. y Vaughan, G. (2010). Psicología Social. Madrid: Editorial Medica Panamericana.

18. Isen, A. y Levin, P. (1972). Effect of feeling good on helping: cookies and kindness. Journal of Personality and Social Psychology, 21, 384-388. http://dx.doi.org/10.1037/ h0032317

19. Javaloy, F., Rodríguez-Carballeira, A., Cornejo, J. M. y Espelt, E. (1998). Felicidad y conducta prosocial. Revista de Psicología Social 13, 205-210. http://dx.doi. org/10.1174/021347498760350696

20. Libet, B. (1985). Unconscious cerebral initiative and the role of conscious will in voluntary action. Behavioral and Brain Sciences, 8, 529-566. http://dx.doi.org/10.1017/ $\underline{\mathrm{S} 0140525 \mathrm{X} 00044903}$

21. Muñoz, J. (2013). Neurofilosofía y libre albedrío. Revista Internacional de Filosofia, 59, 57-70.

22. Nesse, R.M. y Williams G.C. (1996). Why we get sick: the new science of darwinian medicine. Vintage Books. New York: Vintage Books.

23. Oerlemans, W. y Bakker, A. (2014). Why extraverts are happier: A day reconstruction study. Journal of Research in Personality. 50, 11-22. http://dx.doi.org/10.1016/j. irp.2014.02.001

24. Stillman, T., Baumeister, R. y Mele, A. (2011). Free will in everyday life: Autobiographical accounts of free and unfree actions. Philosophical Psychology. 24, 381-394. http:// dx.doi.org/10.1080/09515089.2011.556607

25. Torregrosa, J. (1984). Emociones, sentimientos y estructura social. A E. Crespo i J. Torregrosa, (1984) Estudios básicos de psicología social (pp. 185-200). Barcelona: Hora.

Fecha de recepción: 26 de marzo, 2015

Fecha de recepción de la versión modificada: 25 de julio, 2015

Fecha de aceptación: 30 de julio, 2015 